\title{
Actitudes, evaluación y racionalidad
}

\author{
EDUARdo CRESPo SuÁREZ \\ Universidad Complutense
}

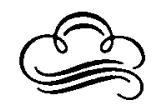

Siempre me ha llamado la atención el limitado y escaso papel que el concepto de racionalidad juega en una ciencia que, como la Psicología Social, está directamente vinculada a los procesos de construcción social del conocimiento. Así, por ejemplo, en un conocido manual de Psicología Social Cognitiva (Eiser, 1986) se dice que «el término "racional" es peligroso, y puede usarse en diferentes contextos para implicar "racionable", "ético", "que implica pensamiento consciente", "sensible" y cosas asím (p. 214). A continuación manifiesta el autor que «la noción de racionalidad, tal como la usan los psicólogos, ... es un concepto básicamente utilitario, basado en la comparación de beneficios y costes esperados» (Ibid.). Esta opinión refleja, creo, el modo de pensar y de investigar más habitual en nuestra disciplina que, interesado por el rigor, se empantana e incomoda con referencias a la conciencia subjetiva, a la normatividad social y a los procesos sociales de construcción del significado. El concepto inmanentista de cognición ha sustituido, como dice Graumann (1988), al concepto relacional de conciencia y conocimiento, y el concepto de actitud ha perdido su vinculación con la acción para entenderse como opinión y evaluación subjetivas.

Mi propósito con este escrito es sondear, aunque sea de forma elemental, la posibilidad y potencial productividad de una consideración de la racionalidad como elemento explicativo dentro de una teoría social de las actitudes. Considero que un concepto no exclusivamente utilitario o instrumental de la racionalidad puede ser de utilidad para comprender ciertos desarrollos de la dinámica actitudinal, en concreto, aquellos en que es posible pensar que cambiamos de actitud porque somos argumentalmente convencidos.

Aunque el concepto de racionalidad sea habitualmente ajeno a la teorización psicosocial, no es, en absoluto, extraño a la tradición científica de las ciencias sociales. La racionalidad viene vinculada tanto a la pretensión de conocimiento científico social como a las posibles caracterizaciones de su objeto de conocimiento, como cualidad de la acción humana. Siendo un concepto complejo y polisémico - Lenk (1988), por ejemplo, describe veintiún usos diferentes, dentro de la epistemología científica, del concepto de racionalidad-, cuya complejidad sería pretencioso y absurdo querer aquí desentrañar, considero que tiene como característica central una referencia a la posibilidad de la legitimación de la acción, tanto instrumental como discursiva.

Dirección del autor: Dpto. Psicología Social 
Según Habermas (1987), el problema de la racionalidad se le plantea a toda sociología a tres niveles: metateórico, metodológico y empírico. En estos mismos tres niveles podríamos considerar que se plantea en la Psicología Social. A nivel metateórico, en tanto en cuanto se reflexiona sobre las implicaciones de los conceptos de acción por los que se guía esta ciencia; en el terreno metodológico, en cuanto se cuestionan los procesos o modos de comprensión de su ámbito objetual, y en el empírico en la medida en que la interacción social es entendida como potencial proceso de comunicación y racionalización. Es en este último nivel en el que creo que se puede plantear una reflexión sobre la racionalidad/irracionalidad de los procesos actitudinales.

En el sentido en que aquí se utiliza, el concepto de racionalidad hace referencia a una cualidad de la actividad humana según la cual ésta se hace susceptible de evaluación en virtud de criterios en los que participa el propio actor, y que le posibilitan para dar razón de sus actividades, acciones o actitudes. La racionalidad, tal como aquí la entiendo, tiene que ver con la legitimación de la acción y con los criterios pertinentes para ello. Legitimar o dar razón de los actos es considerado, pues, como un hecho social, sustentado en criterios y abierto, por ello, a la crítica. Este dar razón o explicación de los actos y actitudes constituye, justamente, el objeto de la teoría de la atribución, tal como la entendió, por ejemplo, Heider (1958), para quien la explicación cotidiana de la acción estaba íntimamente vinculada al establecimiento de responsabilidades en el ámbito de las relaciones interpersonales.

Racionalidad, según esta acepción, supone un modelo implícito de ser humano caracterizado por la reflexividad, agencia y responsabilidad; es decir, por una potencial autonomización tanto en el mundo del pensamiento como en el de la personalidad y el del reconocimiento social. Se trata, por tanto, de un modelo de posibilidad y no de una estruetura necesariamente realizada. Cierto es que la racionalidad no es una característica permanente, tal vez ni siquiera habitual, del hacer humano, y que, como dice Norbert Elías (1982), "toda la historia es hasta hoy, en el fondo, un cementerio de sueños humanos" (p. 32), refiriéndose con ello a las ideologías racionalizantes, pero también es cierto que el sueño de la razón produce monstruos y que a la comprensión de algunos de esos monstruos, como el fascismo, el racismo, el fanatismo o la xenofobia se han dedicado algunos de los estudios más interesantes en el campo de las actitudes y la Psicología Social. Lo que me interesa resaltar, en definitiva, es la posibilidad de considerar las actitudes, entendidas como procesos de significación evaluativa o toma de postura respecto a otros significativos, como procesos sociales susceptibles de enjuiciamiento y eventualmente necesitados de legitimación o justificación.

\section{ALGUNAS CARACTERISTICAS DEL CONCEPTO DE ACTITUD: ACTI- TUD Y EVALUACION.}

El concepto clásico de actitud contiene como núcleo definicional principal una referencia a la evaluación. La mayoría de las definiciones existentes de actitud hacen referencia al proceso de evaluación de un objeto, con el que se interactúa o se puede interactuar. Así, por ejemplo, en la revisión que $M$. Brewster Smith (1968) hacía sobre el tema del cambio de actitudes para la Enciclopedia 
Internacional de Ciencias Sociales, manifestaba que «los psicólogos han investigado bajo esta rúbrica [de actitud] las orientaciones favorables o desfavorables hacia objetos y temas sociales consensualmente definidos» (p. 458). Esta idea se mantiene veinte años más tarde: «Existe una idea generalizada de que una actitud tiene, en su base, un elemento de evaluación» (Zanna y Rempel, 1988: 315). De hecho, estos autores definen la actitud como «la categorización de un estímulo a lo largo de una dimensión evaluativa» (Ibid. 319). Ajzen (1988) considera, igualmente, que las actitudes son constructos hipotéticos que se manifiestan en respuestas que son «de naturaleza evaluativa y dirigidas a un objeto dado» (p. 7). Para Eiser (1986), asimismo, las actitudes son «experiencias subjetivas de algún tema u objeto en términos de una dimensión evaluativa» (p. 11).

Las anteriores referencias - y otras muchas que podrían añadirse - parecen reflejar un acuendo bastante general sobre el carácter evaluativo de las actitudes, que para muchos es su característica definicional. El prototipo de esta evaluación es la reacción afectiva de agrado/desagrado. Triandis (1974), por ejemplo, después de revisar diversas definiciones de actitud dice: «Una definición que incluye muchas de las ideas principales usadas por los teóricos en actitudes, sería la siguiente: "Una actitud es una idea cargada de emotividad que predispone una clase de acciones a una clase particular de situaciones sociales"» (pp. 2-3). La evaluación, entendida como emotividad, se remite a la vivencia subjetiva de "estados agradabes o desagradables» (Triandis, 1974: 11). Esta identificación de evaluación y afecto ya la mantenía Thurstone (1931), quien concebía las actitudes como el efecto a favor o en contra de un objeto psicológico. Aunque esta equiparación de afecto y evaluación no es compartida por todos - Zanna y Rempel (1988), por ejemplo, consideran la evaluación como un proceso de comparación-, sí es ampliamente admitida en las explicaciones teóricas y aún más en las investigaciones empíricas.

Las actitudes, así entendidas, suelen considerarse como mediadoras de la acción, actuando como una predisposición comportamental adquirida hacia un objeto social (Allport, 1935; Newcomb, Turner y Coverse, 1965; Rokeach, 1968; Ajzen, 1987; Ostrom, 1984). Esta evaluación actitudinal, en tanto se entiende como predisposición, se pretende que sea predictiva, aunque la evidencia empírica en este sentido sea poco concluyente (Hill, 1981).

Este concepto de actitud, como predisposición afectivo-evaluativa, que con diferentes matices mantienen la mayoría de los investigadores, se sustenta en una noción objetivante de las relaciones interpersonales. Tal objetivación se caracteriza por una separación analítica del sujeto y el objeto. El carácter procesual e interactivo de las relaciones interpersonales es reducido, bien a una psicología taxonómica o a una psicología diferencial (Moscovici, 1972). La unidad de análisis, aun cuando se plantee en términos formales de interacción, es el sujeto individual, considerado como nivel autónomo, bien de relaciones con el exterior, bien de procesamiento de información (Graumann, 1988). Esta concepción del sujeto como fundamento autónomo de rasgos estables es lo que caracteriza una concepción identitaria de la persona. Las actitudes se consideran, según esto, como una propiedad del sujeto en su relación con el medio objetivado, bien sea éste personal o institucional. Este objeto es considerado social por su carácter público, y no por su estructura significativa. El establecimiento de una dicotomía entre el sujeto que evalúa y el objeto, ante el que reacciona y actúa, implica una concepción asocial y psicologizante del proceso de evaluación al que la actitud se refiere. Por ello, no es extraño que la relación entre actitud 
y conducta $o$ acción se suela entender exclusivamente en términos de antecedente/consecuente, de causa/efecto, que hipotéticamente posibilitar la predicción.

El cambio de actitud se entiende, según esto, como resultante de la influencia social, es decir, como efecto de la actividad de un agente externo, fuente de la influencia. Al entenderse el cambio de actitud como proceso de influencia, considerada ésta como inducción unidireccional al cambio, parece presuponerse la existencia de una especie de resistencia natural al cambio de actitud. Cuando éste se da, lo es como resultante del ejercicio de poder. Cambiar de actitud es, pues, un acto de sometimiento a una fuente ajena de poder. Es ésta, en realidad, una concepción conservadora de la dinámica actitudinal, en la que la acción se entiende de forma instrumental y el cambio como violentamiento. Esta concepción actitudinal va pareja de un interés preferencial por el refinamiento técnico y la predicción comportamental. Ahora bien, como dice $\mathrm{E}$. Zaretsky, editor de la versión de 1984 de la obra de W. Thomas y F. Znaniecki, «El Campesino Polaco», «conforme los sociólogos desarrollaron nuevos y más sofisticados medios de analizar y medir las actitudes... el concepto de actitud quedó reducido a opiniones subjetivas, perdiendo tanto la profundidad como la orientación a la acción que antes le había caracterizado. El esfuerzo por captar la lógica interna o significado de la acción... fue progresivamente perdiéndose en una búsqueda casi fetichista de lo cuantitativo» (p. 27).

\section{Actitudes y acción}

Frente a las teorizaciones en que se considera a la actitud como un proceso previo e independiente de la acción, existen algunas explicaciones psicosociales en las que la actitud juega un papel teórico vinculado a la acción y, más en concreto, a la interacción simbólica. Frente al modelo identitario y objetivamente, la actitud ha sido concebida por cierta Psicología Social como un elemento de la acción o como acción misma (Lalljee, Brown y Ginsburg, 1984; Kelman, 1974, 1984). En este sentido, la recuperación de algunas propuestas clásicas puede posibilitar el trabajo con explicaciones cuyo fundamento no sea exclusivamente el de la racionalidad estratégica è instrumental. De este modo podríamos comprender que - al menos potencialmente- es posible cambiar de actitud como consecuencia del diálogo y la argumentación.

William Thomas y Florian Znaniecki (1919), por ejemplo, pretendían dar una explicación de los procesos sociales de transformación que ocurrían en la comunidad rural polaca cuando emigraba a los Estados Unidos. Para ello, elaboraron una teoría no determinista, fundamentada en un concepto propositivo de acción, poniendo de manifiesto el juego mutuo de factores objetivos y subjetivos (valores y actitudes) que se dan en dichos procesos sociales.

En la famosa «Nota metodológica», introductoria de «El Campesino Polaco», definen los conceptos principales a los que recurren en su explicación, y principalmente el de actitud:

«Por actitud - dicen - entendemos un proceso de conciencia individual que determina la actividad real o posible del individuo en el mundo social.» (p. 58) |.../ «La actitud se distingue de un estado síquico por su referencia a la actividad y, por ende, al mundo social.» (p. 59) /.../ «Cualquier manifestación de la vida consciente de cualquier miembro del grupo es una actitud cuando se le considera en conexión con los valores que constituyen la esfera de experiencia de ese grupo.» (p. 64). 
Para W. Thomas y F. Znaniecki las sociedades desarrolladas cumplen los fines de la regulación social, objeto de su análisis, promoviendo en los individuos «la habilidad para controlar espontáneamente sus propias actividades por medio de reflexión consciente» (p. 14), es decir, por medio de actitudes racionales más que por formas personales y emotivas de control grupal o familiar, propias de las sociedades tradicionales.

George Herbert Mead, por su parte, entiende la actitud como preparación para la acción pero a diferencia de otras explicaciones que se basan en una concepción lineal de la acción - susceptible de ser analizada en términos causa/efecto, antecedentes/consecuentes, variable independiente/dependiente etc.- Mead (1972) recurre a un concepto dialógico de la acción que, cuando ésta es simbólica, se hace reflexivo. La actitud, según ello, es el inicio del acto, donde «las etapas posteriores del acto están presentes en las primeras etapas, no simplemente en el sentido de que están preparadas para ponerse en funcionamiento, sino en el de que sirven para controlar el proceso mismo» (p. 58). Esta presencia del acto como conjunto ya en su inicio y la función de control de la propia acción que esta anticipación tiene, constituye, en mi opinión, uno de los elementos principales del discurso teórico meadiano. El significado de una actitud no viene dado por la supuesta vivencia que exprese, sino por el reconocimiento que recibe, por el significado que se le da cuando se la interpreta. En realidad, las actitudes para Mead sólo existen en la comunicación. El concepto de gesto y el de signo significante o símbolo están íntimamente vinculados al de actitud y en cierto modo se confunden: «El ofrecimiento de una silla a una persona que entra en una habitación es, en sí, un acto de cortesía... y ésa es precisamente la actitud del individuo. Desde el punto de vista del observador, se trata de un gesto.» (Ibid. p. 61).

La comunicación simbólica se caracteriza, para Mead, justamente por la interiorización de las actitudes de los otros miembros de la comunidad, lo cual implica un desconcentramiento o capacidad de ponerse en el lugar de los otros (role-taking), en definitiva, una comunidad de significados.

Al entender de esta forma las actitudes, como parte de la acción comunicativa, se nos abre, creo, una posibilidad de comprensión de alguno de los procesos de construcción significativa de la acción. El estudio de las actitudes se desplaza desde la conciencia a la comunicación y de la identidad trascendental a la interacción discursiva.

Los recientes estudios de pragmática formal realizados por Jürgen Habermas pueden ayudarnos a entender este proceso y permitirnos una teorización de las actitudes en cuanto significados evaluativos o tomas de postura mantenidas en el curso de la acción. La teoría de la acción comunicativa de Habermas, fuertemente enraizada en la teoría de la interacción simbólica de Mead, así como en los criterios weberianos de fundamentación de la autoridad, permite comprender el proceso de interacción social, incluyendo su anticipación y elaboración simbólica como actitud, sin necesidad de entenderlo reductivamente como acción instrumental y el cambio de actitud como proceso exclusivo de influencia. En su teoría se establecen criterios analíticos para distinguir entre la acción teleológica y la acción comunicativa, así como de los procesos de racionalidad que les son inherentes.

Habermas (1989), en las famosas «Christian Gauss Lectures», pronunciadas en la Universidad de Princeton en 1971, plantea una serie de distinciones conceptuales, que en obras posteriores desarrollará de forma más minuciosa. Una 
distinción primera, ya planteada por Weber, es la que se da entre comportamiento y acción. La base de esta distinción es el sentido o significado. Weber (1964) calificaba de conducta «puramente reactiva» a aquella conducta a la que el agente no asocia ningún significado subjetivamente deseado. Habermas (1989) califica, por su parte, como acción al comportamiento intencional, dirigido por reglas: «Las normas tienen un contenido semántico, justamente un sentido que siempre que un sujeto capaz de entenderlo las sigue, se ha convertido en razón o motivo de un comportamiento; y es entonces cuando hablamos de una acción» (p. 21). En una obra posterior, Habermas (1987) entiende por acción una forma de habérsela con las situaciones y dominarlas. Esta acción puede conceptuarse bien como acción teleológica de realización de fines (acción instrumental o estratégica) o como acción comunicativa, en la que «los participantes persiguen de común acuerdo sus respectivos planes de acción sobre la base de una definición común de la situación» (Habermas, 1987, vol II: 180). La racionalidad inherente a cada uno de estos tipos de acción es diferente. En una obra reciente (Habermas, 1990), plantea que «la racionalidad tiene menos que ver con la posesión del saber que con el modo como lo emplean sujetos capaces de lenguaje y de acción.» (p. 71). La racionalidad con arreglo a fines supone el empleo no comunicativo del saber en acciones teleológicas. Este es el tipo de racionalidad subyacente a la mayoría de los modelos de cambio de actitud. Se consideran racionales y, por tanto, estables aquellas actitudes que son funcionales para la consecución de algún tipo de recurso o la satisfacción de alguna necesidad. Habermas, sin embargo, considera que existe una racionalidad inherente al entenderse, a cuyo concepto «subyace intuitivamente la experiencia de la fuerza unificadora sin coacción y fundadora de consenso que lleva en su seno el habla argumentativa.« (Ibid. p. 72). Esta racionalidad no tiene como fundamento la eficacia, que es un criterio propio de la racionalidad con arreglo a fines, sino que se mide «por el plexo que forman las condiciones de validez de los actos de habla, las pretensiones de validez que se entablan con los actos de habla y las razones con que pueden desempeñarse discursivamente tales pretensiones» (Ibid. p. 72).

La teoría habermasiana se enraiza en una teoría sociológica donde es frecuente encontrar una elaborada distinción entre diferentes tipos de comportamientos en virtud de los procesos sociogenéticos que los caracterizan y, por tanto, de los posibles procesos de evaluación y racionalización a que son sometibles. Evaluación y racionalidad son, desde esta perspectiva, conceptos complementarios. La racionalidad apunta a los criterios de aceptabilidad de un comportamiento en virtud de sus características socio-históricas.

Para Max Weber, por ejemplo, el elemento definitorio de la acción humana es el sentido subjetivo que los actores dan a su comportamiento. Este sentido es comprensible - y enjuiciable - en virtud de los posibles y diferentes sistemas de orientación de la acción o criterios de racionalidad. Asimismo, para este autor, la actitud en la acción está vinculada a los distintos sistemas de relación social y de racionalidad. Weber hablaba, en concreto, de racionalidad afectiva y tradicional para referirse a los sistemas sociales de constitución de la acción en sociedades a las que caracteriza como comunidad: «La actitud en la acción social -en el caso particular, por término medio o en el tipo puro (de la comunidad) - se inspira en el sentimiento subjetivo (afectivo o tradicional) de los partícipes de constituir un todo.» (Weber, 1964: 33).

En las sociedades modernas los tipos de motivación son caracterizables, de 
modo general, bien como racionales con arreglo a fines o con arreglo a valores: «Llamamos sociedad a una relación social cuando y en la medida en que la actitud en la acción social se inspira en una compensación de intereses por motivos racionales (de fines o de valores) o también en una unión de intereses con igual motivación.» (Ibid.: 33).

Si conceptuamos las actitudes como un momento de la acción, y ésta es caracterizada por su sentido, parece coherente recuperar para la teoría psicosociológica de las actitudes una reflexión en términos de racionalidad. Hablar de racionalidad en relación con las actitudes supone aceptar que existe algún tipo de estándar de evaluación de éstas, lo que las hace susceptibles de crítica. Supone considerarlas no tanto como una propiedad inmanente a un sujeto ideal cuanto, por su propia significatividad, como un proceso social susceptible de legitimación en sus pretensiones de validez, es decir, susceptible de enjuiciamiento bajo criterios de racionalidad. De hecho, suele aceptarse implícitamente un criterio de racionalidad en gran parte de los estudios sobre actitudes: el criterio derivado de la creencia en un orden natural de los procesos de pensamiento y afección. Un orden básicamente instrumental donde lo irracional es tratado como sesgo, es decir, como perturbación de ese orden universal de la naturalización y donde la desviación de la «normalidad» es considerada como resultado de una interferencia de otros factores, sean éstos conocidos o no.

El lugar social de esta evaluación y legitamación actitudinal es la interacción comunicativa y la argumentación. Las actitudes por su propio carácter comunicacional y lingüístico exigen, por tanto, interpretación, y se hacen acreedoras a la posibilidad de lograr un acuerdo racional en base a la argumentación.

Esta posibilidad de justificación racional del discurso actitudinal se plantea en dos planos. El primero se refiere a la aceptabilidad de relatos por parte de los actores sociales, es decir, a la legitimación cotidiana de las manifestaciones actitudinales. El segundo, a la posibilidad de un enjuiciamiento valorativo y crítico por parte del científico social como supuesto participante privilegiado.

Respecto al primer plano, el de la legitimación cotidiana, pueden ser interesantes, nuevamente, las ideas de Habermas acerca de la acción comunicativa estratégica y la acción comunicativa orientada al entendimiento. En el primer caso, la aceptación de las actitudes ajenas -y por tanto de su versión de la acción - es un hecho - directo o encubierto- de poder. En este sentido, una interesante tarea para una Psicología Social de las actitudes sería el indagar entre otras cosas - en los criterios de aceptabilidad de los relatos explicativos de las actitudes y en los mecanismos retóricos que los fundamentan en una sociedad y momento concretos, lo cual nos permitiria ahondar en el conocimiento del orden lingǘstico y moral de ese grupo social.

En el segundo caso, lo que se plantea es la posibilidad de considerar, frente a la razón del poder, el poder de la razón; poder que se ejerce en una situación de diálogo, de lenguaje ideal, que no irreal o idealista, ya que ese carácter ideal es inherente a la propia naturaleza del lenguaje. Este segundo aspecto tiene que ver con los intereses del conocimiento. Este es un territorio problemático, pues si bien el interés de emancipación, propio del conocimiento crítico, ha inspirado notables investigaciones como es el estudio de la personalidad autoritaria, no es menos verdad que no siempre éstas son sostenibles teórica o metodológicamente. La posibilidad, incluso, de tal saber crítico es puesta en cuestión (ahí está la polémica entre modernidad y postmodernidad). Esta es una cuestión que, de momento, prefiero dejar abierta, aunque reconozco que me resultan atracti- 
vas algunas posiciones como la recientemente defendida por Alain Touraine, en el XII Congreso Mundial de Sociología, sobre la recuperación de un concepto pluralista y no dogmático de modernidad, muy en la línea de lo que Habermas ha caracterizado como pensamiento postmetafísico.

\section{Notas}

1. Según Dumonceaux (1975), en Francia, en el siglo XVII, la palabra «sentiment» era muy cercana en su significado a «opinión» y «avis». El «sentiment» no surge de una fuente propiamente afectiva, en el sentido actual, sino que «expresa de manera inmediata y sin ambigüedad una manera de ver las cosas estrechamente personal, que se traduce a menudo por una toma de posición personal» (p. 448). En un sentido muy similar utiliza el término castellano «sentimiento» Jerónimo de Barrionuevo en sus famosos Avisos de 1956.

2. En un sentido similar al aquí utilizado, Uli Windisch $(1982,1990)$ habla de una pluralidad de formas lógico-discursivas, caracterizadoras de diferentes estructuras socio-cognitivas.

\section{Referencias}

Adorno. T.; Frenkel-Brunswik, E.; Levinson, D., y Sanford, N. (1950), The authoritarian Personality. N. York: Harper.

Ajzen, I (1987) Attitudes, Traits, and Actions: Dispositional prediction of behavior in personality and social psychology. En L. Berkowitz (ed.) Advances in experimental social psychology. vol. 20. San Diego: Academic Press.

AJzen, I., (1988). Attitudes, personality and behavior. Milton Keynes: Open Univ. Press.

Allport, G. W. (1935). Attitudes. En C. Murchison (ed.) A bandbook of socialpsychology. Worcester: Clark University Press.

COOPER, J. B. (1959). Emotions in prejudice. Science 130, 314-18.

Crespo, E. (1981). Teorías de la consistencia cognoscitiva. Madrid: U.C.M.

EIser, J. R. (1986). Social Psychology. Attitudes, cognition and social bebaviour. Cambridge: Cambridge Univ. Press.

ELIAS, N. (1982). Sociología fundamental. Barcelona: Gedisa.

Graumann, C.F. (1988). From knowledge to cognition. En D. Bar-Tal y A. W. Kruglanski (eds.) The social psychology of knowledge. Cambridge: Cambridge Univ. Press.

Habermas, J. (1987). Teoria de la acción comunicativa. 2 vols. Madrid: Taurus.

Habermas. J. (1989). Teoria de la acción comunicativa: Complementos y estudios previos. Madrid: Cátedra

Habermas, J. (1990). Pensamiento postmetafísico. Madrid: Taurus.

HeIDER, F. (1958). The Psychology of Interpersonal Relations. N. York: Wiley.

Hill, R. J. (1981). Attitudes and behavior. En Rosenberg y Turner (eds.) Social Psychology. Sociological perspectives. N. York: Basic Books.

Jones, E. E., y Gerard, H. B. (1967). Foundations of Social Psycbology. N. York: Wiley.

Kelman, H. C. (1974). Attitudes are alive and well and gainfully employed in the sphere of action. American Psychologist. 310-324.

Kelman, H. C. (1984). La influencia social y los nexos entre el individuo y el sistema social; más sobre los procesos de sumisión, identificación e internalización. En J. R. Torregrosa y E. Crespo (eds.) Estudios básicos de psicología Social. Barcelon: Hora.

Lalljee, M.; Brown, L. B., y Ginsburg, G. P. (1984). Attitudes: Disposition, behaviour or evaluation? British Joumal of Social Psychology. 23, 233-244.

LENK, H. (1988). Entre la epistemología y la ciencia social. Barcelona: Alfa.

Mead, G. H. (1972). Espíritu, persona y sociedad. B. Aires: Paidós (3.a edic.).

Moscovici, S. Cociety and Theory in Social Psychology. En J. Israel y H. Tajfel (eds.) The context of Social Psichology. London: Academic Press.

Newcomb, T. M.; Turner, R. H., y Converse, P. E. (1965). Social Psychology. N. York: Holt.

Ostrom, T. M. (1984). The sovereignty of social cognition. En R. S.

WYER y T. K. SRULL (eds.) Handbook of social cognition. Vol. I. Hillsdale, NJ.: Lawrence Erlbaum. 


\section{5}

ROKEACH, M. (1968). Beliefs, attitudes and values. S. Fco. Calif.: Jossey-Bass.

Smith, M. B. (1968). Attitude change. En D. L. Sills (ed.) International Encyclopedia of the Social Sciences. Crowell, Collier MacMillan. Vol. 1.

Thomas, W., y Znaniecki, F. (1919). The Polish Peasant in Europe and America (edic. de Eli Zaretsky) Urbana: University of Illinois Press (1984).

Thurstone, L. L. (1931). The measurement of attitudes. Journal of Abnormal and Social Psycbology, 26, 249-269.

Triandis, H. C. (1974). Actitudes y cambio de actitudes. Barcelona: Toray.

Weber, M. (1964). Economía y sociedad. México: F.C.E.

Zanna, M. P., y Rempel, J. K. (1988) Attidudes: a new look at an old concept. En D. Bar-Tal y A. W. Kruglanski (eds.) The social psychology of knowledge. Cambridge: Cambridge Univ. Press 